\title{
FATORES FAVORÁVEIS À RECUPERAÇÃO DO QUADRO CLÍNICO DE CRIANÇAS ANÊMICAS: UM ESTUDO EXPLORATÓRIO
}

\author{
Favorable factors for recovering clinical features \\ of anemic children: an exploratory study
}

\author{
Juliana Nunes Santos ${ }^{(1)}$, Stela Maris Aguiar Lemos ${ }^{(2)}$, Therezinha Vieira ${ }^{(3)}$, Joel Alves Lamounier ${ }^{(4)}$
}

\begin{abstract}
RESUMO
Objetivo: investigar a existência de aspectos do ambiente familiar de crianças anêmicas que possam ser considerados favoráveis à recuperação do quadro de anemia e ao desenvolvimento da linguagem. Método: trata-se de um estudo de caso de crianças com anemia. As crianças $(n=11)$ foram submetidas anteriormente ao tratamento clínico e avaliação da linguagem. Após 12 meses, foram reavaliadas nos aspectos clínicos e da linguagem, acrescentando-se a investigação dos recursos do ambiente familiar. Deste grupo, foram selecionados três casos que refletem diferentes evoluções do quadro clínico da anemia e do desenvolvimento da linguagem, a fim de explorar as contribuições do ambiente familiar na melhora desses aspectos. Resultados: a comparação das variáveis entre as crianças que obtiveram melhora ou não no tratamento medicamentoso mostra diferença estatisticamente nos valores de hemoglobina e tempo de aleitamento materno $(p<0,05)$. A análise dos três casos revela que a situação ambiental pode ser agravante do quadro clínico da criança. Conclusões: a qualidade dos recursos domiciliares, o tempo de aleitamento materno e as práticas parentais em relação à criança podem ter contribuído para a adequada recuperação da criança nos aspectos focalizados.
\end{abstract}

DESCRITORES: Anemia; Desenvolvimento da Linguagem; Criança; Família

(1) Fonoaudióloga; Doutora em Saúde da Criança e do Adolescente pela Faculdade de Medicina da Universidade Federal de Minas Gerais (FM-UFMG); Professora Adjunto do Curso de Fonoaudiologia da Universidade Federal de Minas Gerais (UFMG), Belo Horizonte, Minas Gerais, Brasil.

(2) Fonoaudióloga; Doutora em Distúrbios da Comunicação Humana e Professora Adjunto do curso de graduação em Fonoaudiologia da Universidade Federal de Minas Gerais (UFMG), Belo Horizonte, Minas Gerais, Brasil.

(3) Psicóloga; Doutora em Psicologia pela Universidade de São Paulo; Professora Adjunto aposentada do Departamento de Psicologia da Universidade Federal de Minas Gerais (UFMG), Belo Horizonte, Minas Gerais, Brasil.

(4) Médico Pediatra; Professor Titular do Departamento de Pediatria e Coordenador do Programa de Pós-graduação em Saúde da Criança e do Adolescente da Faculdade de Medicina da Universidade Federal de Minas Gerais (UFMG), Belo Horizonte, Minas Gerais, Brasil; Doutor em Saúde Pública pela Universidade da Califórnia em Los Angeles (UCLA-EUA).

Conflito de interesses: inexistente

\section{INTRODUÇÃO}

No ambiente familiar, vínculos frágeis ${ }^{1}$, contextos pouco estimuladores ${ }^{2}$ e interações sociais prejudicadas $^{3}$ podem resultar em obstáculos para o desenvolvimento infantil, especialmente no que diz respeito ao desenvolvimento de linguagem. Tais fatores acrescentam riscos a mais para crianças que já se encontram vulneráveis por algum tipo de enfermidade, como a anemia que atinge mais de $50 \%$ das crianças entre seis meses e cinco anos de idade nos países em desenvolvimento ${ }^{4}$.

No mundo, a anemia afeta cerca 1,62 bilhão de pessoas, o que corresponde a $24,8 \%$ da população mundial. No continente americano, estima-se que 23,1 milhões de crianças pré-escolares sejam anêmicas ${ }^{5}$. No Brasil, a prevalência da doença em menores de seis anos de idade é de $53 \%$, confirmando valores estimados pela $\mathrm{OMS}^{6}$. 
Em termos de saúde pública, a reconhecida relevância do combate à anemia decorre não apenas da magnitude da ocorrência desse tipo de adoecer, mas, principalmente, das repercussões negativas que ocasiona no desenvolvimento psicomotor, cognitivo, social e da linguagem dos indivíduos $^{7-9}$. Tal combate pode se dar através de medidas preventivas, ou medicamentosas, caso a doença já tenha se instalado. Medidas preventivas, como provisão de alimentos fortificados com ferro para segmentos vulneráveis, diversificação da dieta alimentar da população, programas de educação alimentar e incentivo ao aleitamento materno, só podem efetivar-se por meio de políticas governamentais de saúde pública que propiciem os recursos necessários para tal ${ }^{10-12}$. Quando são necessários tratamentos medicamentosos, há que se conhecer a etiologia da anemia, para proporcionar maior eficácia desse tratamento. Contudo, estudos têm demonstrado que na faixa etária de pré-escolares em $80 \%$ dos casos a anemia é causada pela carência de ferro no organismo ${ }^{13}$.

Quando se trata de anemia ferropriva, os sais de ferro têm sido tradicionalmente utilizados, sendo os esquemas de suplementação diária e semanal os mais discutidos na literatura recente $5,10,14,15$. Entretanto, são relatadas na literatura grandes dificuldades de adesão aos programas baseados na suplementação medicamentosa, visto que grande parte das crianças abandona a intervenção antes do período previsto ${ }^{16}$. Os efeitos colaterais do medicamento (intolerância gastrintestinal, alterações na cor e consistência das fezes e impregnação de ferro nas fraldas e nos dentes), o tempo prolongado e o custo também são fatores limitantes à referida adesão ${ }^{10,14,16}$.

No estudo de base realizado com essas crianças ${ }^{17}$ comparou-se o desenvolvimento de linguagem entre anêmicos e não anêmicos. Constataram-se diferenças com significância estatística entre os grupos avaliados, com pior desempenho de linguagem para as crianças anêmicas. Estas últimas foram tratadas com sulfato ferroso durante 12 semanas, sendo que $67 \%$ melhoraram com o tratamento. Este estudo, assim como outras pesquisas da área, revela uma forte associação entre a anemia e as alterações de linguagem ${ }^{7,8}$.

Pode ser, porém, que crianças com anemia que simultaneamente vivam em ambientes pouco estimuladores do desenvolvimento da linguagem tenham menos chances de superar alterações dessa linguagem, o que pode, em princípio, acarretar inúmeros prejuízos para aprendizagens futuras. Dentre tais ambientes, podem-se destacar o ambiente familiar e instituições de cuidado e educação, como as creches ou escolas infantis.
É nesses dois tipos de ambientes que a criança passa a maior parte do seu dia. Considerando que a linguagem se desenvolve em diferentes contextos de uso, mediante as trocas que se dão no interior da cultura ${ }^{3}$, se esses contextos limitam ou diminuem a probabilidade de ocorrência de tais trocas, é provável também que mesmo crianças que tenham superado a anemia continuem apresentando distúrbios de linguagem, devido a fatores biológicos consequentes da deficiência de ferro ${ }^{18}$ e/ou por não disporem de oportunidades suficientes para se exercitar no uso da linguagem ${ }^{19}$.

Como no estudo de base ${ }^{17}$ não foram explorados tais aspectos socioculturais, este estudo prolonga o anterior, na medida em que busca aproximar-se dessa questão, ainda que de uma forma exploratória, dada a sua complexidade e relevância. Nesse sentido, parte-se do pressuposto que a análise de ambientes familiares pode apontar fatores suscetíveis de favorecer ou dificultar o desenvolvimento da linguagem. Assim, este estudo pretende investigar a existência de aspectos do ambiente familiar de crianças anêmicas que possam ser considerados favoráveis à recuperação do quadro de anemia e do desenvolvimento da linguagem.

\section{MÉTODO}

Este estudo integra um projeto de pesquisa mais abrangente, longitudinal, de avaliação das repercussões da anemia no desenvolvimento da criança. Foi aprovado pelo Comitê de Ética em Pesquisa da Universidade Federal de Minas Gerais (UFMG), sob o protocolo 380/05.

Neste estudo, de caráter quantitativo e qualitativo, propõe-se uma abordagem dialética: saúde e doença são percebidas como fenômenos clínicos e sociológicos vividos culturalmente, uma vez que o corpo humano está atravessado pelas determinações das condições, situações e estilos de vida ${ }^{20}$.

As análises se inspiraram no estudo de caso. Metodologicamente, os estudos de caso evidenciam o contexto em que uma intervenção ocorreu, o rumo de um processo em curso e a maneiras de interpretá-lo buscando o sentido e a relevância de algumas situações-chave nos resultados de uma intervenção ${ }^{20}$.

\section{Amostra}

O universo do presente estudo compõe-se de crianças de uma creche pública, na faixa etária de 51 a 71 meses. Como critérios de inclusão considerou-se a presença do quadro de clínico de anemia no ano de $2006{ }^{17}$ e a realização do tratamento clínico com sulfato ferroso durante 12 semanas. Foram excluídas as crianças que não 
foram localizadas após 12 meses do término do tratamento medicamentoso.

Das 11 crianças em estudo, 8 (72\%) são do gênero masculino e todas são provenientes de camadas de baixo nível socioeconômico, já que a creche atende somente crianças mediante comprovação de renda dos pais.

Foram selecionados do universo em estudo 3 (três) casos que refletem diferentes evoluções do quadro clínico da anemia e do desenvolvimento da linguagem, a fim de explorar as contribuições do ambiente familiar na melhora desses aspectos.

\section{Procedimentos}

Na primeira etapa do estudo, as crianças foram avaliadas quanto à anemia e ao desenvolvimento da linguagem ${ }^{17}$. Posteriormente, as mesmas receberam tratamento à base de sulfato ferroso durante 12 semanas.

Na segunda etapa do estudo, 12 meses após o término do tratamento medicamentoso, as crianças foram reavaliadas quanto ao desenvolvimento da linguagem, bem como foram feitas novas medidas de hemoglobina e realizada a investigação dos recursos do ambiente familiar.

\section{Avaliação da anemia}

As crianças foram avaliadas por meio de punção digital para a determinação do nível de hemoglobina $(\mathrm{Hg})$, utilizando-se o espectrofotômetro de alta precisão HemoCue@ . Com uma microcuveta, obteve-se o volume preciso de sangue em contato com quantidade exata de reagente seco. A microcuveta foi, então, inserida no HemoCue@ , determinando o valor de hemoglobina entre 15 e 45 segundos ${ }^{21}$.

Para caracterizar o estado de anemia, consideraram-se os valores estabelecidos pela Organização Mundial de Saúde (2008): hemoglobina $<11,0 \mathrm{~g} / \mathrm{dl}$ para crianças abaixo de cinco anos e $<11,5 \mathrm{~g} /$ dl para crianças de 5 a 11 anos ${ }^{5}$. A este valor foi acrescentado 0,3 (pela variabilidade do aparelho), para se obter mais sensibilidade no diagnóstico. Considerou-se a anemia como anemia ferropriva, pois na faixa etária avaliada, $80 \%$ das anemias são causadas pela deficiência de ferro ${ }^{13}$, sendo essa a mais comum na população de pré-escolares brasileiros ${ }^{6}$.

As crianças portadoras de anemia foram submetidas à terapêutica em dose única semanal de 4,0 $\mathrm{mg} / \mathrm{Kg} /$ dia durante 12 semanas ${ }^{22}$. Depois de concluído o tratamento, todos participantes foram avaliados, verificando-se aumento médio nos níveis de hemoglobina. Contudo, 5 crianças ainda apresentavam valores de hemoglobina compatíveis com a anemia. Na avaliação subsequente, 12 meses depois de concluído o tratamento, a anemia ainda estava presente nas mesmas 5 crianças.

\section{Avaliação da linguagem}

Em 2006, as crianças foram avaliadas segundo critérios definidos por Chiari, utilizando-se o Roteiro de Observação de Comportamentos de crianças de 0 a 6 anos ${ }^{23}$. O desenvolvimento da linguagem de cada um dos participantes foi observado quanto aos aspectos comunicativos de recepção e emissão da linguagem. Os registros das respostas referentes aos comportamentos esperados para cada idade foram feitos em fichas individuais, assinalando-se "Sim" para a presença e "Não" para a ausência. Foram criados índices de desempenho (ID) a fim de qualificar as respostas das crianças ${ }^{17}$.

Em 2008, transcorridos 12 meses do término do tratamento, as crianças foram reavaliadas quanto ao desenvolvimento da linguagem, utilizando-se as tarefas do instrumento de Avaliação de Desenvolvimento de Linguagem (ADL), proposto para identificar alterações na aquisição e desenvolvimento da linguagem ${ }^{24}$.

A ADL é um instrumento clínico, validado, eficiente para avaliar o conteúdo e a estrutura da linguagem na faixa etária de 1 ano a 6 anos e 11 meses. A administração da ADL é individual e avalia os domínios receptivos e expressivos da linguagem. O conteúdo da linguagem é verificado por meio de tarefas concentradas nos conceitos de quantidade, qualidade (adjetivos), relação espacial, temporal e sequência. A estrutura da linguagem é avaliada por meio de tarefas referentes à morfologia e à sintaxe ${ }^{24}$.

A mudança do instrumento de avaliação de linguagem entre as etapas do estudo foi realizada de modo a contemplar uma faixa etária maior de crianças (até 6 anos e 11 meses de idade) e não implicou prejuízos para o estudo, já que a ADL, assim como o Roteiro de Observação, avalia os domínios receptivos e expressivos da linguagem. Além disso, as análises seguiram o delineamento do tipo transversal comparativo (anêmicos e não anêmicos) em cada momento do estudo em separado.

\section{Investigação dos recursos do ambiente familiar}

$\mathrm{Na}$ segunda etapa da pesquisa (ano de 2008), o inventário de recursos do ambiente familiar (RAF) ${ }^{25}$ foi aplicado às mães ou responsáveis pela criança, sob a forma de entrevista semiestruturada, no momento em que levavam ou buscavam seus filhos na creche.

O referido instrumento, inicialmente projetado para investigar os recursos do ambiente familiar que podem contribuir para o aprendizado 
acadêmico nos anos do ensino fundamental, sofreu adaptações, a fim de investigar o ambiente familiar de crianças da educação infantil. Cada tópico foi apresentado à mãe ou responsável oralmente, tendo o examinador a liberdade de parafrasear 0 conteúdo da questão quando houvesse dificuldade de compreensão por parte da pessoa entrevistada.

Este instrumento, composto de 10 tópicos, avalia três domínios elencados pela autora: recursos que promovem processos proximais; atividades que sinalizam estabilidade na vida familiar; e práticas parentais que promovem a ligação família-escola. Estes dois últimos domínios foram adaptados para contemplar a realidade da educação infantil.

O domínio de recursos que promovem processos proximais compreende as seguintes áreas de recursos e atividades: i) participação em experiências estimuladoras do desenvolvimento, como passeios e viagens; ii) oportunidades de interação com os pais; iii) disponibilidade de brinquedos e materiais que apresentam desafio ao pensar; iv) disponibilidade de livros, jornais e revistas; v) uso adequado do tempo livre; e vi) acesso a atividades programadas de aprendizagem; são representados pelas questões de números 1 a 7 .

No domínio de atividades previsíveis que sinalizam algum grau de estabilidade na vida familiar estão incluídas áreas de recursos tais como rotinas e reuniões regulares da família, representada pelas questões de números 9 e 10. A questão 9 sofreu modificações, sendo que não foram investigadas as atividades "almoçar" e "fazer a lição de casa", já que as crianças almoçam na creche os cinco dias letivos da semana e não levam tarefas para casa.

As práticas parentais que promovem a ligação família-escola englobam indicadores de envolvimento direto dos pais na vida escolar, como participação nas reuniões e verificação do material escolar da criança, itens contemplados na questão número 8. As perguntas sobre supervisão da lição de casa e do estudo das provas e sobre acompanhamento de notas e frequência da criança às aulas não foram realizadas, já que não se aplicam à realidade das crianças da creche.

A pontuação bruta em cada um dos 10 tópicos seguiu os critérios descritos pela autora ${ }^{25}$.

\section{Análise dos dados}

Para a entrada, o processamento e a análise quantitativa dos dados foi utilizado o software SPSS - versão 14.0. Para fins de análise descritiva, foi feita distribuição de frequência das variáveis categóricas envolvidas na avaliação da linguagem em estudo e análise das medidas de tendência central e de dispersão das variáveis contínuas. Foi testada a normalidade da distribuição das variáveis.
Utilizou-se o teste Exato de Fisher para verificar diferenças entre as proporções e o teste Kruskal Wallis na análise de variáveis contínuas com nível de significância de 95\% ( $p \leq 0,05)$.

Para fins de análise qualitativa, foi utilizada a técnica de análise de conteúdo temática, constituída de três etapas ${ }^{20}$. Na primeira etapa fez-se a escolha dos casos que constituíram o corpus do estudo, em que se buscou atender às normas de validade qualitativa de representatividade, homogeneidade, pertinência e exaustividade. A segunda etapa se caracterizou pela exploração do material, em que se buscou encontrar categorias e expressões significativas, em função do conteúdo em estudo. E, por fim, na terceira etapa realizou-se o tratamento dos resultados obtidos e interpretação.

\section{RESULTADOS}

Primeiramente, serão descritas as características dos sujeitos que compõem o universo em estudo. As crianças, suas mães e os recursos do ambiente familiar são analisados e comparados segundo a evolução do tratamento medicamentoso da anemia (Tabela 1).

A comparação das variáveis entre as crianças que obtiveram melhora ou não no tratamento medicamentoso mostra que os grupos diferiram estatisticamente nos valores de hemoglobina e tempo de aleitamento materno. Das crianças que não melhoraram com o tratamento medicamentoso todas são do gênero masculino e possuem distúrbio de linguagem.

As características das crianças e suas mães segundo a evolução ao tratamento medicamentoso da anemia estão representadas na Figura 1. Esta também destaca as diferenças entre as crianças que melhoraram ou não com o tratamento medicamentoso da anemia.

Para o aprofundamento da análise das questões referentes ao ambiente familiar das crianças, foram selecionados três casos que contêm as características essenciais do universo estudado, a saber:

- Caso 1: criança com insucesso no tratamento medicamentoso da anemia e desenvolvimento da linguagem alterado.

- Caso 2: criança com sucesso no tratamento medicamentoso da anemia e desenvolvimento da linguagem alterado.

- Caso 3: criança com sucesso no tratamento medicamentoso da anemia e desenvolvimento da linguagem normal.

Os casos, com suas respectivas caracterizações do ambiente e da estrutura familiar, assim como as práticas parentais em relação à criança podem ser visualizados na Figura 2. 
Tabela 1 - Distribuição das características de onze crianças, suas mães e o ambiente familiar, apresentadas segundo a evolução do quadro de anemia da criança no período de 2006 a 2008

\begin{tabular}{|c|c|c|c|c|c|c|c|c|c|}
\hline \multirow{3}{*}{\multicolumn{2}{|c|}{ CARACTERÍSTICAS }} & \multicolumn{6}{|c|}{ Tratamento da anemia } & \multirow{3}{*}{$\begin{array}{l}\text { Kruskall } \\
\text { Wallis }\end{array}$} & \multirow{3}{*}{$\mathbf{P}$} \\
\hline & & \multicolumn{3}{|c|}{ Bem sucedido $(n=6)$} & \multicolumn{3}{|c|}{ Mal sucedido $(n=5)$} & & \\
\hline & & $\mathbf{P}_{25}$ & mediana & $\mathbf{P}_{75}$ & $\mathbf{P}_{25}$ & mediana & $\mathbf{P}_{75}$ & & \\
\hline \multicolumn{2}{|c|}{ Idade das crianças (meses) } & 55,0 & 67,5 & 69,5 & 56,5 & 62,0 & 66,5 & 1,21 & 0,27 \\
\hline \multicolumn{2}{|c|}{ Hemoglobina (g/dL) } & 11,7 & 12,6 & 13,6 & 9,9 & 10,3 & 11,2 & 7,5 & ${ }^{\star} 0,006$ \\
\hline \multicolumn{2}{|c|}{ Anos de estudos das mães } & 5,7 & 8,0 & 9,5 & 4,5 & 8,0 & 11,0 & 0,009 & 0,93 \\
\hline \multicolumn{2}{|c|}{ Aleitamento materno (meses) } & 5,3 & 24,0 & 40,5 & 2,5 & 5,0 & 5,5 & 3,77 & ${ }^{*} 0,05$ \\
\hline \multicolumn{2}{|c|}{ Escore total do RAF } & 39,5 & 44,5 & 53,0 & 36 & 50 & 61 & 0,13 & 0,71 \\
\hline \multicolumn{2}{|c|}{ Escore: acesso a passeios } & 1,0 & 3,5 & 5,3 & 2,0 & 3,0 & 7,0 & 0,86 & 0,35 \\
\hline \multicolumn{2}{|c|}{$\begin{array}{l}\text { Escore: atividades compartilhadas } \\
\text { com os pais }\end{array}$} & 2,0 & 3,0 & 4,3 & 1,0 & 4,0 & 6,0 & 0,03 & 0,85 \\
\hline \multicolumn{2}{|c|}{$\begin{array}{l}\text { Escore: disponibilidade de } \\
\text { brinquedos }\end{array}$} & 5,0 & 7,5 & 10,0 & 6,0 & 9,0 & 13,0 & 0,68 & 0,40 \\
\hline \multicolumn{2}{|c|}{ Escore: disponibilidade de livros } & 1,0 & 1,0 & 2,0 & 1,5 & 2,0 & 5,5 & 3,1 & 0,08 \\
\hline \multicolumn{2}{|c|}{$\begin{array}{l}\text { Escore: práticas parentais que } \\
\text { promovem a ligação família-escola }\end{array}$} & 7,7 & 8,0 & 8,0 & 4,5 & 7,0 & 8,0 & 2,53 & 0,11 \\
\hline \multirow{2}{*}{\multicolumn{2}{|c|}{ Escore: rotina de atividades diárias }} & 8,5 & 9,5 & 10,3 & 7,5 & 9,0 & 11,0 & 0,21 & 0,64 \\
\hline & & & & & & \multicolumn{3}{|c|}{ Teste Exato de Fisher } & $\mathbf{P}$ \\
\hline \multirow{2}{*}{ Linguagem } & Normal & \multicolumn{2}{|r|}{3} & \multicolumn{2}{|c|}{0} & \multirow{2}{*}{\multicolumn{2}{|c|}{4,57}} & & $0.07^{*}$ \\
\hline & Alterada & \multicolumn{2}{|r|}{3} & \multicolumn{2}{|c|}{5} & & & & \\
\hline \multirow{2}{*}{ Gênero } & Masculino & & 3 & \multicolumn{2}{|c|}{5} & \multirow{2}{*}{\multicolumn{2}{|c|}{1,98}} & & \multirow{2}{*}{0,18} \\
\hline & Feminino & & 3 & & & & & & \\
\hline
\end{tabular}

Legenda: Teste Kruskall Wallis; Nivel de significância de 95\% ( $p \leq 0,05)$;

$\mathrm{P}_{25}=$ Percentil 25; $\mathrm{P} 75=$ Percentil 75.

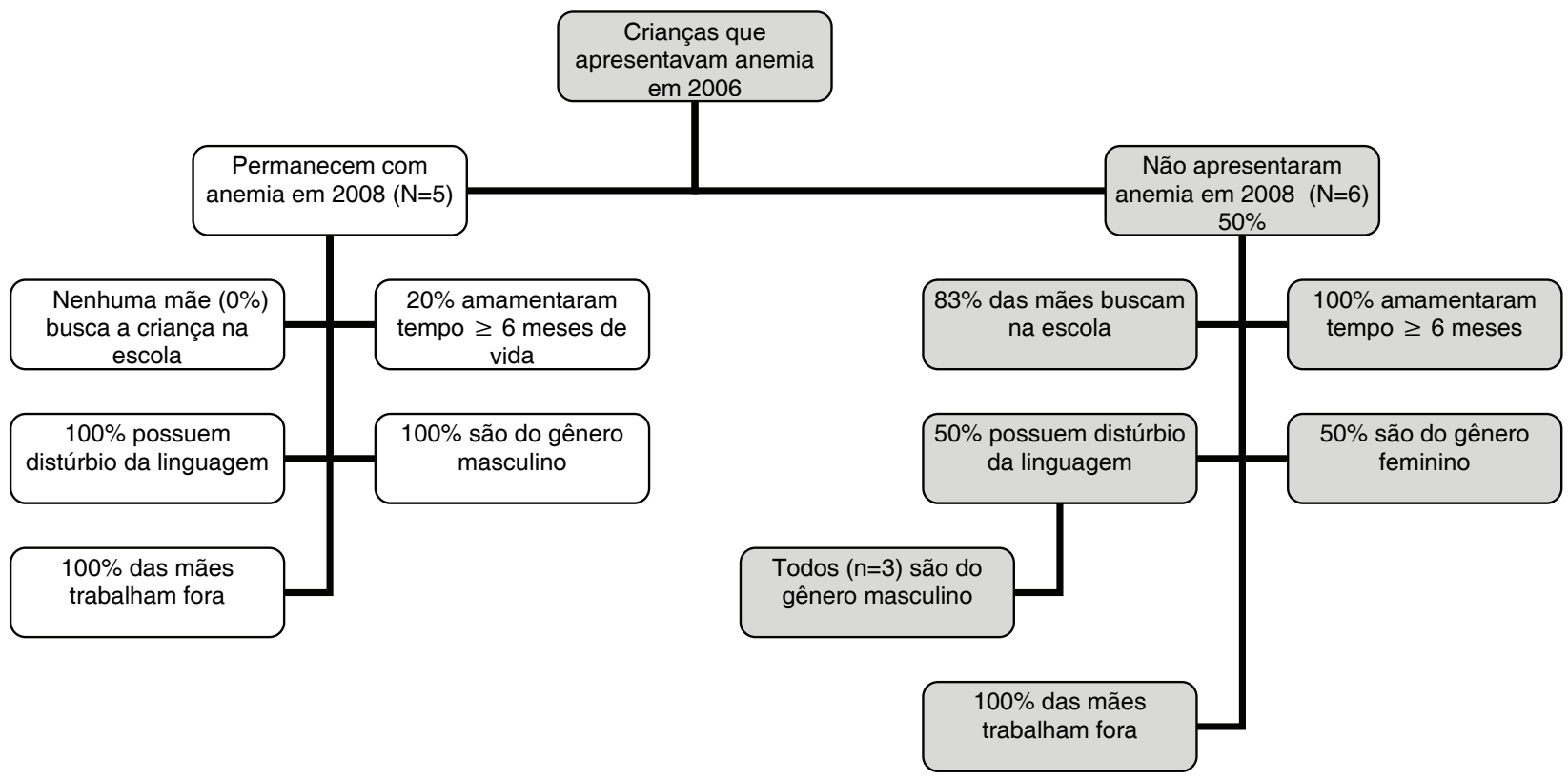

Figura 1 - Características das crianças e suas mães segundo a evolução do quadro clínico da anemia 


\begin{tabular}{|c|c|c|c|}
\hline & Caso 1 & Caso 2 & Caso 3 \\
\hline $\begin{array}{l}\text { Características } \\
\text { gerais da } \\
\text { criança }\end{array}$ & $\begin{array}{l}\text { Gênero masculino; } 4 \text { anos e } \\
\text { três meses. } \\
\text { Quadro clínico de anemia. } \\
\text { Distúrbio do } \\
\text { desenvolvimento da } \\
\text { linguagem. }\end{array}$ & $\begin{array}{l}\text { Gênero masculino; } 4 \text { anos e } \\
7 \text { meses. } \\
\text { Ausência do quadro clínico } \\
\text { de anemia. } \\
\text { Distúrbio do } \\
\text { desenvolvimento da } \\
\text { linguagem. }\end{array}$ & $\begin{array}{l}\text { Gênero feminino com idade } \\
\text { de } 5 \text { anos e } 3 \text { meses. } \\
\text { Ausência do quadro clínico } \\
\text { de anemia. } \\
\text { Desenvolvimento da } \\
\text { linguagem adequado. }\end{array}$ \\
\hline $\begin{array}{l}\text { Envolvimento } \\
\text { dos pais com a } \\
\text { criança }\end{array}$ & $\begin{array}{l}\text { Mãe ausente permanecendo } \\
\text { até } 4 \text { meses sem ver o filho, } \\
\text { criado com a bisavó. } \\
\text { Não existe atividade } \\
\text { estimuladora de linguagem } \\
\text { partilhada com a criança } \\
\text { dentro de casa. }\end{array}$ & $\begin{array}{l}\text { Envolvimento limitado a } \\
\text { assistir programas de } \\
\text { televisão. } \\
\text { Ausência de trocas } \\
\text { interativas - histórias e/ou } \\
\text { conversas sobre o dia da } \\
\text { criança ou outros assuntos. } \\
\text { Ausência de interação } \\
\text { partilhada em atividades } \\
\text { domésticas ou em } \\
\text { brincadeiras. }\end{array}$ & $\begin{array}{l}\text { Envolvimento dos pais com } \\
\text { a criança por meio de } \\
\text { atividades partilhadas como } \\
\text { brincadeiras, programas de } \\
\text { televisão infantil, conversa } \\
\text { sobre o dia na escola e } \\
\text { realização de atividades } \\
\text { domésticas. }\end{array}$ \\
\hline $\begin{array}{l}\text { Práticas } \\
\text { parentais que } \\
\text { promovem a } \\
\text { ligação família } \\
\text { escola }\end{array}$ & $\begin{array}{l}\text { A mãe permanece ausente e } \\
\text { a bisavó realiza as } \\
\text { atividades rotineiras } \\
\text { necessárias ao } \\
\text { encaminhamento da criança } \\
\text { para a creche. }\end{array}$ & $\begin{array}{l}\text { A mãe é a responsável por } \\
\text { acompanhar as atividades } \\
\text { escolares da criança nos } \\
\text { momentos em que está em } \\
\text { casa. }\end{array}$ & $\begin{array}{l}\text { A mãe e a avó se revezam } \\
\text { no acompanhamento das } \\
\text { atividades escolares da } \\
\text { criança. }\end{array}$ \\
\hline $\begin{array}{l}\text { Momentos de } \\
\text { família reunida }\end{array}$ & $\begin{array}{l}\text { Momentos esporádicos em } \\
\text { passeios aos finais de } \\
\text { semana. }\end{array}$ & $\begin{array}{l}\text { Assiste televisão à noite em } \\
\text { família e toma café da } \\
\text { manhã juntos. }\end{array}$ & $\begin{array}{l}\text { Assiste televisão à noite em } \\
\text { família e no jantar. Às vezes, } \\
\text { passeia nos finais de } \\
\text { semana. }\end{array}$ \\
\hline $\begin{array}{l}\text { Estrutura } \\
\text { familiar }\end{array}$ & $\begin{array}{l}\text { Mora com duas pessoas } \\
\text { idosas (bisavós). }\end{array}$ & $\begin{array}{l}\text { Mora com a avó, mãe (que } \\
\text { trabalha fora) e dois irmãos. }\end{array}$ & $\begin{array}{l}\text { Mora com a avó, mãe (que } \\
\text { trabalha fora) e dois irmãos. }\end{array}$ \\
\hline $\begin{array}{l}\text { Acesso a } \\
\text { material } \\
\text { impresso }\end{array}$ & $\begin{array}{l}\text { Não tem acesso a qualquer } \\
\text { material impresso, a não ser } \\
\text { o material escolar. }\end{array}$ & $\begin{array}{l}\text { Acesso a revistas de } \\
\text { notícias e livros escolares. }\end{array}$ & $\begin{array}{l}\text { Acesso a revistas de } \\
\text { notícias e de esportes, e } \\
\text { livros; além dos escolares, } \\
\text { livros infantis, religiosos e } \\
\text { enciclopédias. }\end{array}$ \\
\hline $\begin{array}{l}\text { Atividades } \\
\text { independentes } \\
\text { da mediação do } \\
\text { adulto }\end{array}$ & $\begin{array}{l}\text { Criança brinca somente } \\
\text { dentro de casa com } \\
\text { brinquedos, em sua maioria } \\
\text { de estimulação motora. } \\
\text { Brinca de videogame e } \\
\text { assiste televisão. } \\
\text { Não brinca na rua. }\end{array}$ & $\begin{array}{l}\text { Criança brinca na rua. } \\
\text { Possui brinquedos que } \\
\text { favorecem o } \\
\text { desenvolvimento motor, } \\
\text { brinquedos pedagógicos } \\
\text { (números, letras, cores, } \\
\text { tamanhos e formas) e } \\
\text { imaginativos (de faz de } \\
\text { conta). }\end{array}$ & $\begin{array}{l}\text { Criança brinca dentro de } \\
\text { casa, lê livros e gibis, ouve } \\
\text { rádio e assiste televisão. } \\
\text { Possui brinquedos que } \\
\text { favorecem o } \\
\text { desenvolvimento motor, } \\
\text { brinquedos pedagógicos } \\
\text { (números, letras, cores, } \\
\text { tamanhos e formas, lousa, } \\
\text { giz), imaginativos (de faz de } \\
\text { conta) e jogos de regras. }\end{array}$ \\
\hline $\begin{array}{l}\text { Acesso a } \\
\text { passeios ou } \\
\text { outras } \\
\text { atividades } \\
\text { externas }\end{array}$ & $\begin{array}{l}\text { Visitas a parentes e amigos, } \\
\text { sem participar de atividades } \\
\text { programadas externas. }\end{array}$ & $\begin{array}{l}\text { Visitas a parentes e amigos, } \\
\text { sem participar de atividades } \\
\text { programadas externas. }\end{array}$ & $\begin{array}{l}\text { Visitas a parentes e amigos, } \\
\text { com idas a lanchonetes e } \\
\text { parques, sem participar de } \\
\text { atividades programadas } \\
\text { externas. }\end{array}$ \\
\hline
\end{tabular}

Figura 2 - Características de três das crianças e seus ambientes familiares, com diferentes evoluções no quadro clínico da anemia um ano após tratamento medicamentoso 
A criança "1" interage regularmente apenas com dois adultos, já idosos; a mãe parece ausente. A criança aparenta ter poucas oportunidades de conversar, seja em casa ou na rua, com adultos ou com outras crianças. Dispõe de poucos brinquedos e material impresso. A linguagem a que tem acesso mais frequentemente é a da televisão. O interesse da família pela escola parece restringir-se ao essencial. Os dados arrolados na Figura 1 apontam para um ambiente pouco estimulador do ponto de vista do desenvolvimento da linguagem. Trata-se de criança que, além da anemia, vivencia um ambiente de escasso estímulo social e intelec tual.

A criança "2" participa de um grupo familiar maior, comparativamente à primeira criança (mãe, avó e dois irmãos). A criança parece ter mais oportunidades de interações, quer em casa, quer na rua, relativamente à criança 1 . Suas trocas interativas parecem ocorrer predominantemente em períodos determinados do dia. Do ponto de vista de recursos materiais, também é um pouco mais favorecida (brinquedos, material impresso) e desfruta de um ambiente um pouco mais enriquecido do que o da criança 1 no que toca a situações propiciadoras do desenvolvimento da linguagem. A mãe se mostra mais presente no que toca às atividades escolares. Mesmo assim, a criança apresenta um distúrbio de linguagem.

A criança " 3 ", do ponto de vista do grupo familiar, não difere da criança 2 (mãe, avó e dois irmãos). No entanto, os dados arrolados na Figura 2 indicam que ela vivencia situações de trocas verbais mais variadas no âmbito familiar e extrafamiliar e dispõe de recursos materiais mais variados. A família parece mais interessada na oferta desses recursos (já que os adquire para a criança). Parece também interessar-se pela leitura (pelo tipo de material impresso disponível no ambiente doméstico); fato que neste caso, provavelmente, acaba propiciando modelos de sujeitos leitores à criança.

\section{DISCUSSÃO}

Ao abordar a questão do sucesso ou insucesso do tratamento medicamentoso, considera-se que estes fenômenos se projetam em dois planos distintos: o biológico e o sociocultural. No âmbito sociocultural, podem-se ainda delimitar unidades menores para efeito de estudo, por exemplo, contextos familiares e escolares. Nesse sentido, justifica-se a opção metodológica dialética adotada no presente estudo (relação entre dados biológicos com socioculturais), a fim de se alcançar um maior entendimento dos fatores susceptíveis na recuperação da anemia e no desenvolvimento da linguagem. Os sujeitos deste estudo que obtiveram formas de evolução diferentes no tratamento da anemia são crianças pertencentes a famílias com práticas, costumes, rotinas e crenças próprias. Elas provêm de uma condição social menos favorecida e frequentam uma instituição social de cuidado e de educação (a creche), com suas condutas, valores e significados também próprios.

A análise dos dados da Tabela 1 mostra que as crianças com evoluções diferentes ao tratamento da anemia apresentam muitas características semeIhantes, tais como idade, escolaridade materna e sob um prisma mais quantitativo até mesmo um ambiente familiar semelhante. No entanto, ao comparar o tempo de aleitamento materno total entre os dois grupos, evidencia-se diferença de 19 meses entre as medianas, diferença com significância estatística, com menor tempo de amamentação no grupo de crianças que obtiveram insucesso terapêutico ao tratamento da anemia. Isso leva a questionar se a amamentação no início da vida teria um efeito protetor para a criança, de modo que seu organismo responderia melhor ao tratamento de fortificação. A literatura indica que sim. O leite materno, do ponto de vista nutricional, é reconhecido como o alimento adequado para a criança nos primeiros meses de vida, não só por disponibilizar energia, macro e micronutrientes, mas também pela proteção que confere contra doenças ${ }^{11}$. Este fator protetor do aleitamento materno, especialmente contra a anemia ${ }^{10}$, foi confirmado em estudos da população brasileira de latentes e pré-escolares ${ }^{26-28}$. Esses estudos concluíram que o aleitamento materno exclusivo até os seis meses de idade e a manutenção do leite materno a partir dessa idade, associados aos alimentos complementares quali e quantitativamente adequados contribuem para o aumento dos níveis de hemoglobina. Nesta pesquisa, a manutenção do aleitamento materno nas crianças não impediu que as crianças desenvolvessem a anemia, mas parece tê-las protegido de quadros mais graves da doença, assim como parece ter contribuído para que respondessem positivamente ao tratamento.

Estudos sobre os efeitos da terapia com ferro no desenvolvimento da criança mostram que os piores escores nos testes de desenvolvimento podem persistir mesmo após tratamento ${ }^{8,31}$; outros estudos revelam que pode haver melhora no desenvolvimento com o tratamento à base de sais de ferro ${ }^{32}$. Nesta pesquisa, $50 \%$ das crianças que melhoraram os níveis de hemoglobina pós-tratamento medicamentoso persistiram com o distúrbio de linguagem.

A mãe que amamenta por mais tempo provavelmente estabelece um vínculo mais fortalecido com seu filho e uma melhor relação de apego ${ }^{29}$. Isso pode levar a mãe a cuidar mais da saúde 
da criança, realizando o tratamento e a estimulando mais nos aspectos do desenvolvimento. $O$ bebê, neste caso, pode tornar-se mais saudável sob diversos aspectos, quer físicos, emocionais ou cognitivos. Em relação ao desenvolvimento da linguagem, é possível perceber que todas as crianças que persistem com o quadro de anemia apresentam distúrbio do desenvolvimento da linguagem (Tabela 1). Estas mesmas crianças apresentaram piores habilidades comunicativas de recepção e emissão da linguagem quando comparadas com as crianças não-anêmicas antes do tratamento ${ }^{17}$. A literatura mostra que crianças anêmicas constituem uma população de risco para pior desenvolvimento cognitivo, motor, linguístico e socioemocional em relação às não anêmicas, podendo apresentar efeitos tardios da anemia na infância que se prolongam até a vida adulta ${ }^{7,8,30}$.

Um segundo indicador de vinculo mãe - criança se apoia no fato de as mães buscarem seus filhos na creche. Esta informação parece estar relacionada com uma maior disponibilidade da mãe para com a criança e melhor vínculo mãe - filho, os quais também seriam indicados pela manutenção do aleitamento materno por um tempo superior a 6 meses para estas crianças com resposta adequada ao tratamento. De fato, as atitudes dos pais têm um importante papel na qualidade do crescimento de seus filhos e um impacto em seu desenvolvimento cognitivo e linguístico ${ }^{2,33}$. Neste estudo, os resultados também sugerem que as práticas parentais positivas em relação à criança e a ligação família - escola se relacionam com melhor resposta ao tratamento e menor repercussão negativa no desenvolvimento da linguagem, com um valor de " $p$ " clinicamente significativo $(p=0,09)$.

Ao comparar os resultados do RAF entre os dois grupos de crianças que obtiveram ou não êxito no tratamento medicamentoso da anemia, a princípio, não são observadas diferenças com significância estatística (Tabela 1). Uma das explicações para este resultado pode ser o tamanho da amostra, já que, ao analisar os três casos separadamente (Figura 2), fica explícita a presença diferenciada de estímulos no ambiente das três crianças, evidenciando que a terceira criança teve um ambiente mais estimulador. Segue-se daí a hipótese de que este tipo de ambiente tem contribuído para o desenvolvimento da linguagem da criança em detrimento daqueles que se apresentaram para as crianças 1 e 2, favorecendo, talvez, a maior adesão ao tratamento por parte da família, aparentemente mais interessada na criança. Isso está de acordo com o que aponta a literatura especializada a respeito: constata-se uma associação entre a qualidade do ambiente e o desenvolvimento das crianças ${ }^{2}$ No entanto, pode-se ressaltar a importância de fatores biológicos ${ }^{17}$ e sociais ${ }^{1,8}$ para esse mesmo desenvolvimento infantil.

A análise aprofundada dos três casos revela aspectos importantes do ambiente das crianças e suas famílias. No caso 1 , é possível divisar o enfraquecimento das situações interativas adulto - criança e criança - criança no domicílio, aspecto que vai se destacando cada vez mais do caso 2 para o 3, com reversão progressiva desta característica ambiental. No caso 3 também é possível perceber maior diversidade de situações que favorecem o desenvolvimento da linguagem, como as possibilidades de contato com diferentes portadores de texto e objetos lúdicos. Vários autores discutem a importância da interação social para a aquisição de linguagem, especialmente as relações da criança com a mãe, as quais representam um sistema dinâmico, que contextualiza as interações, sendo que ambos os membros da díade contribuem com suas experiências e conhecimentos para o curso da interação ${ }^{3}$. Cabe ressaltar neste estudo que as variações nas situações interativas identificadas para as três crianças mostram também diferenças na forma e na frequência com que elas vivenciam aspectos particulares da linguagem. Além disso, deve-se considerar a influência dos aspectos biológicos inerentes ao quadro clínico da anemia, os quais, segundo estudos, propiciam uma menor interação mãe - filho ${ }^{34}$, e a influência do gênero, já que a criança do caso 3 é uma menina.

Os recursos favorecedores do desenvolvimento de linguagem também apresentaram grandes diferenças entre os casos. A criança 3 , que obteve adequada resposta ao tratamento clínico e apresenta desenvolvimento adequado, possui em seu domicílio a maior variedade de brinquedos e, mais que isso, maior partilha de conhecimentos com os adultos que cuidam dela, os quais lhe permitem desenvolver vários aspectos cognitivos, sobretudo, a linguagem. A criança 2 possui um número mais limitado de recursos. A criança 1 realiza brincadeiras predominantemente motoras, tendo se observado a escassez de recursos em seu domicílio. A oferta de recursos e a interação social no ambiente familiar tornam esse ambiente mais estimulador $e$ mais propício ao desenvolvimento das habilidades comunicativas e cognitivas. Tais achados estão em concordância com a literatura, que mostra associações entre a estimulação no ambiente doméstico e o desenvolvimento cognitivo infantil ${ }^{2}$.

Assim, é possível verificar que no caso 1 a situação ambiental constitui um agravante do quadro clínico da criança, que possui a anemia e o distúrbio de linguagem. No caso 2, o ambiente oferece poucos recursos favorecedores do desenvolvimento 
da linguagem e no caso 3 o ambiente é favorecedor desse desenvolvimento. Tais observações, em principio, poderiam explicar, mesmo que parcialmente, as diferentes recuperações das crianças ao quadro da anemia, sugerindo que o ambiente pode ser um fator determinante no sucesso de algumas intervenções terapêuticas. No entanto, não se pode ignorar, presentemente, a questão de gênero, nas suas relações com o desenvolvimento da linguagem, conforme assinalado a seguir.

Neste estudo, a maioria das crianças é do gênero masculino (72\%). Das 5 que não obtiveram êxito no tratamento medicamentoso, todas são meninos e das 3 meninas avaliadas todas apresentaram adequada evolução no tratamento medicamentoso, com repercussão positiva no desenvolvimento da linguagem. Contudo, o êxito no tratamento medicamentoso da anemia, concomitantemente ao melhor desenvolvimento da linguagem para crianças do sexo feminino, em comparação com aquelas do sexo masculino, não pode ser a única explicação para as diferenças encontradas. Há, pelo menos, duas outras ordens de fatores a se considerar. Em primeiro lugar, a literatura revela que, normalmente, as meninas se desenvolvem mais no plano da linguagem do que os meninos, podendo o atraso da linguagem ocorrer três vezes mais em crianças do gênero masculino do que no feminino ${ }^{35}$. Em segundo lugar, as análises mostram que as diferenças nas práticas parentais e em aspectos do ambiente familiar das três crianças analisadas revelaram-se mais favoráveis à menina do que aos meninos. Pode ser, portanto, que variáveis do ambiente familiar interajam com a variável gênero, influenciando tanto o desenvolvimento da linguagem quanto a evolução no tratamento da anemia. Assim, os dados sugerem que ser do gênero feminino e vivenciar determinado tipo de práticas parentais e ou com um ambiente mais estimulador contribui para a recuperação do quadro de anemia, diminuindo o risco de alterações no desenvolvimento da linguagem para as meninas.

Não foi possível, neste estudo, aprofundar a análise relativa às diferenças de gênero em interação com variáveis do ambiente familiar em face do tratamento da anemia e de suas repercussões no desenvolvimento da linguagem, tendo em vista que o tamanho da amostra inviabilizava análises estatísticas apropriadas. Sugere-se que esta questão venha a ser retomada em investigações posteriores.

\section{CONCLUSÃO}

O estudo realizado indica que a qualidade dos recursos do ambiente familiar, o tempo de aleitamento materno e as práticas parentais positivas em relação à criança podem ter contribuído para a adequada recuperação da criança anêmica nos aspectos clínicos e do desenvolvimento da linguagem.

Um ambiente familiar estimulador parece estar relacionado com a recuperação adequada da criança no quadro clínico da anemia e no que diz respeito ao desenvolvimento da criança.

\begin{abstract}
Purpose: to investigate the existence of familial environmental aspects of anemic children that may be regarded as favorable for the recovery of anemia and the language development. Method: this is a case study of anemic children. The children $(n=11)$ were previously submitted to clinical treatment and language assessment. After 12 months, children were reassessed in clinical and language aspects, adding investigation of Home Environment Resources Scale. From this group, three cases were selected, reflecting different evolutions in clinical features' anemia and language development in order to explore the contributions of the familial environment in the improvement of those aspects.

Results: the comparison of the variables between the children who had gotten improvement or not in the clinical treatment showed to be differing statistically as for the values of hemoglobin and maternal breastfeeding's period $(p<0.05)$. The analysis of the three cases shows that the environmental situation can aggravated the child's clinical features. Conclusion: the quality of the familial resources, the time of maternal breastfeeding and the parental practices in relation to the child might have contributed to the child's appropriate recovery in the focused aspects.
\end{abstract}

KEYWORDS: Anemia; Language Development; Child; Family 


\section{REFERÊNCIAS BIBLIOGRÁFICAS}

1. Bradley RH, Corwyn RF. Socioeconomic Status and Child Development. Annual Review of Psychology 2002; 53: 371-99.

2. Maria-Mengel MRS, Linhares MBM. Fatores de risco para problemas de desenvolvimento infantil. Rev. Latino-Am. 2007; 15: 837-42.

3. Borges LC, Salomão NMR. Aquisição da linguagem: considerações da perspectiva da interação social. Psicol. Reflex. Crit 2003; 16: 327-36.

4. Walker SP, Wachs TD, Gardner JM, Lozoff B, Wasserman GA, Pollitt E, Carter JA; International Child Development Steering Group. Child development: risk factors for adverse outcomes in developing countries. Lancet. 2007; 369:145-57.

5. Organização Mundial de Saúde (OMSa). Worldwide prevalence of anaemia 1993 - 2005: WHO global database on anaemia. Geneva, World Health Organization; 2008. [cited 2009 Fev 13]. Available from: http://whqlibdoc.who.int/ publications/2008/9789241596657_eng.pdf

6. Jordão RE, Bernardi JLD, Barros Filho AA. Prevalência de anemia ferropriva no Brasil: uma revisão sistemática. Rev. paul. pediatr 2009; 27 : 90-8.

7. McCann JC, Ames BN. An overview of evidence for a causal relation between iron deficiency during development and deficits in cognitive or behavioral function. Am J Clin Nutr. 2007; 85(4):931-45.

8. Lozoff B, Jimenez E, Smith JB. Double burden of iron deficiency in infancy and low socioeconomic status: a longitudinal analysis of cognitive test scores to age 19 years. Arch Pediatr Adolesc Med 2006;160:1108-13.

9. Beard JL. Why iron deficiency is important in infant development. J Nutr 2008; 138(12):2534-6.

10. Organização Mundial de Saúde (OMS). Iron deficiency anaemia: Assessment, Prevention, and Control. A guide for programme managers. Geneva. United Nations Children's Fund/ United Nations University/ World Health Organization; 2001. [cited 2009 Jul 01]. Available from: http://www.who.int/ nutrition/publications/micronutrients/anaemia_iron_ deficiency/WHO_NHD_01.3/en/index.html

11. Organização Mundial de Saúde (OMS). Learning from large-scale community-based programmes to improve breastfeeding practices. Geneva, World Health Organization, United Nations Children's Fund/ UNICEF, Academy for Educacional development/ AED; 2008. [cited 2009 Ago 07]. Available from: http://www.who.int/nutrition/ publications/infantfeeding/9789241597494/en/ index.html
12. Alderman $\mathrm{H}$, Linnemayr $\mathrm{S}$. Anemia in low-income countries is unlikely to be addressed by economic development without additional programs. Food Nutr Bull. 2009; 30(3):265-9.

13. Asobayire FS, Adou P, Davidsson L, Cook JD, Hurrel RF. Prevalence of iron deficiency with and without concurrent anaemia in population groups with prevalence of malaria and other infections: a study in Côte d'Ivoire. American Journal of Clinical Nutrition 2001; 74:776-82.

14. Sociedade Brasileira de Pediatria (SBP). Anemia Carencial Ferropriva. Rio de Janeiro. Departamento Científico de Nutrologia da Sociedade Brasileira de Pediatria; 2007. [cited 2009 Mar 25]. Available from: http://www.sbp.com.br/img/documentos/doc_ anemia_carencial_ferropriva.pdf

15. Engstrom EM, Castro IRR, Portela M, Cardoso LO, Monteiro CA. Efetividade da suplementação diária ou semanal com ferro na prevenção da anemia em lactentes. Rev. Saúde Pública 2008; 42: 786-95.

16. Torres MAA, Sato K, Juliano Y, Queiroz SS. Terapêutica com doses profiláticas de sulfato ferroso como medida de intervenção no combate à carência de ferro em crianças atendidas em unidades básicas de saúde. Rev. Saúde Pública 1994; 28: 410-5.

17. Santos JN, Rates Silmar PM, Lemos SMA, Lamounier JA. Anemia em crianças de uma creche pública e as repercussões sobre o desenvolvimento de linguagem. Rev Paul Pediatr 2009; 27: 67-73.

18. Lozoff B, Georgieff MK. Iron deficiency and brain development. Semin Pediatr Neurol. 2006; 13:158-65.

19. Black MM, Walker SP, Wachs TD, Ulkuer N, Gardner JM, Grantham-McGregor S, Lozoff $B$, Engle PL, de Mello MC. Policies to reduce undernutrition include child development. Lancet. 2008;371(9611):454-5.

20. Minayo MCS. O desafio do conhecimento pesquisa qualitativa em saúde. 11aㅡ ed. São Paulo: Hucitec; 2008.

21. Mills AF, Meadows N. Screening for anaemia: evaluation of a haemoglobinometer. Archives of Disease in Childhood 1989; 64: 1468-71.

22. Capanema FD. Estudo comparativo de eficácia terapêutica: dose única semanal $x$ dose convencional diária de sulfato ferroso na anemia ferropriva [tese de doutorado]. Belo Horizonte (MG): Faculdade de Medicina, Universidade Federal de Minas Gerais; 2006.

23. Chiari BM, Basilio CS, Nakagawa EA, Cormedi MA, Silva NSM, Cardoso RM, Parreira VEW. Proposta de sistematização de dados da avaliação fonoaudiológica através da observação 
de comportamentos de criança de 0 a 6 anos. PróFono Rev Atual Cient 1991; 3:29-36.

24. Menezes MLM. A construção de um instrumento para avaliação do desenvolvimento da linguagem: idealização, estudo piloto para padronização e validação [Tese de doutorado]. Rio de Janeiro (RJ): Fundação Oswaldo Cruz, FIOCRUZ; 2003.

25. Marturano EM. O inventário de recursos do ambiente familiar. Psicol. Reflex. Crit 2006; 19: 498-506.

26. Konstantyner T, Taddei JAAC, Oliveira MN, Palma D, Colugnati FAB. Riscos isolados e agregados de anemia em crianças frequentadoras de berçários de creches. J. Pediatr. 2009; 85(3): 209-16.

27. Konstantyner TT, Carrazedo JAA, Palma D. Fatores de risco de anemia em lactentes matriculados em creches públicas ou filantrópicas de São Paulo. Rev. Nutr 2007; 20: 349-59.

28. Duarte LS, Fujimori E, Minagawa AT, Schoeps FA, Montero RMJM. Aleitamento materno e níveis de hemoglobina em crianças menores de 2 anos em município do estado de São Paulo, Brasil. Rev. Nutr 2007; 20(2):149-57.

29. Jansen J, Weerth C, Riksen-Walraven. Breastfeeding and mother-infant relationship - A review. Developmental Review 2008; 28:503-21.
30. Benton D; ILSI Europe a.i.s.b.I. The influence of children's diet on their cognition and behavior. Eur J Nutr. 2008;47 Suppl 3:25-37.

31. Eickmann SH, Brito CMM, Lira PIC, Lima MC. Effectiveness of weekly iron supplementation on hemoglobin concentration, nutritional status and development of infants of public daycare centers in Recife, Pernambuco State, Brazil. Cad Saúde Pública 2008; 24: S303-11.

32. Antunes H, Gonçalves S, Teixeira-Pinto A, CostaPereira A, Tojo-Sierra R. Anemia por Deficiência de ferro no Lactente- Resultados preliminares do desenvolvimento aos cinco anos. Acta Med Port 2005; 18: 261-6.

33. Lunkenheimer ES, Dishion TJ, Shaw DS, Connell AM, Gardner F, Wilson MN, Skuban EM. Collateral Benefits of the Family Check-Up on Early Childhood School Readiness: Indirect Effects of Parents' Positive Behavior Support. Developmental Psychology 2008; 44:1737-52.

34. Corapci F, Radan AE, Lozoff B. Iron deficiency in infancy and mother-child interaction at 5 years. $J$ Dev Behav Pediatr. 2006; 27:371-8.

35. Vitto MMP, Feres MCLC. Distúrbios da comunicação oral em crianças. Medicina (Ribeirão Preto) 2005; 38: 229-34.
http://dx.doi.org/10.1590/S1516-18462010005000121

RECEBIDO EM: 07/04/2010

ACEITO EM: 28/07/2010

Endereço de correspondência:

Autor: Juliana Nunes Santos

Rua Coronel Pedro Jorge, ํํ 170 apto 201

Bairro Prado - Belo Horizonte - Minas Gerais

CEP: 30410-350

E-mail: jununessantos@yahoo.com.br 